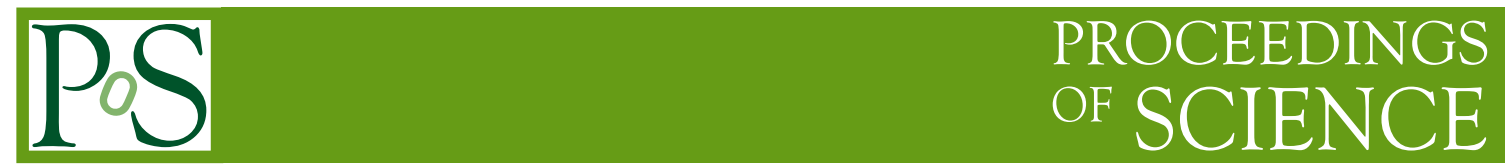

\title{
Noncommutative Gerbes
}

\author{
Paolo Aschieri* \\ Dipartimento di Scienze e Tecnologie avanzate, Universitá del Piemonte Orientale, \\ Viale T. Michel 11, 15121, Alessandria, Italy, and \\ INFN, Sezione di Torino, gruppo collegato di Alessandria, Italy \\ E-mail: aschierieto.infn.it
}

\begin{abstract}
We review the construction of the Seiberg-Witten map (SW map) from commutative to noncommutative gauge theories. We then consider it in the global context of line bundles and thus recall the definition of noncommutative line bundles. We show how to glue togheter noncommutative bundles and thus define noncommutative gerbes. Examples arising from deformation quantization of twisted Poisson structures are presented.
\end{abstract}

Corfu Summer Institute on Elementary Particles and Physics - Workshop on Non Commutative Field Theory and Gravity

September 8 - 12, 2010

Corfu, Greece

* It is a pleasure to thank the organizer of the workshop for the fruitful and pleasant atmosphere. This paper is based on the collaboration [1] with I. Baković, B. Jurco and P. Schupp. 


\section{Introduction}

Gerbes are a higher version of line bundles (bundles with fiber $\mathbb{C}$ ). In electromagnetism we have a gauge potential $A$ and a field strenght $F$. The geometry underlying electromagnetism is that of a line bundle with connection $A$ and curvature $F$. In higher electromagnetism we have a gauge potential two form $B$ and a field strength 3-form $H$. The geometry underlying higher electromagnetism is that of a gerbe with curving $B$ and curvature $H$. In this talk we generalize the notion of gerbe to the noncommutative case. We work in the context of deformation quantization.

There are quite some motivations for this investigation. i) Noncommutative gauge theory has proven useful in the construction of effective $D$-brane actions in a Neveu-Schwarz background $B$-field that is constant $(d B=0)$. We address the more general case where we have a global 3form $H$ and locally $H=d B$. This should lead to a noncommutative description of $D$-branes in a background $H$ that is nonvanishing and even topologically nontrivial (because $H$ is closed but not necessarily exact). ii) Noncommutative gerbes provide further examples of noncommutative geometric structures. While this could be per se interesting we find relevant that our constructuion succesfully tests noncommutative line bundles as building blocks for noncomutative gerbes. iii) Noncommutative gerbes provide examples of quantization of antisymmetric tensors that fail to be Poisson tensors. When the two form $B$ is closed and nondegenerate we have a symplectic structure. Its quantization leads to a well defined associative $\star$-product. When $H=d B \neq 0$ it is usually believed that it is necessary to relax associativity of the corresponding (would be) $\star$ product. We will see that this is not the case if $H$ can be associated with a twisted Poisson tensor, an antisymmetric tensor that fails to be Poisson because of the nonvanishing of $H$ (see 6.2). Indeed in this evenience one can still construct a true Poisson tensor and an associated $\star$-product. If more in general $d H=0$ but is not exact we have to deal with $\star$-products for each open patch where $H$ is exact. It is the noncommutative gerbe geometry that captures the global structure of these local $\star$-products. iv) noncommutative gerbes provide examples of nonabelian gerbes. We therefore have a higher version of the statement that noncommutative $U(1)$ gauge theory is a nonabelian gauge theory.

In this paper we choose to give an overwiew of the many different stept that lead to the construction of noncommutative gerbes in deformation quantization. We refer to the original paper [1] for more details and the notion of noncommutative connection. The purpose here is to emphasize which are the main ingredients and the basic ideas that lead to our results. The notion of noncommutative gerbe in deformation quantization is here also presented slightly more abstractly.

We start in Section 2 by recalling the properties of the Seiberg-Witten map (SW map) between commutative and noncommutative gauge theories $[2,3]$ This map does not only relate commutative $(U(1))$ infinitesimal gauge transformations to infinitesimal noncommutative ones. It also relates the corresponding finite gauge transformations. Finite gauge transformations are associated with transition functions of the underlying bundle, this leads to the notion of noncommutative line bundles [4]. This is the content of Section 3

In Section 4 we then explain, following Hitchin [5], how commutative abelian gerbes can be realized via line bundles. In Section 5 we then use noncommutative line bundles to construct noncommutative gerbes. 
In the last section we provide an example of noncommutative gerbes arising from quantization of twisted Poisson structures.

While the main idea inspiring our construction is that of the Seiberg-Witten map, the definition of noncommutative line bundle can be given abstractly and independently from the notion of SW map. Similarly our definition of noncommutative gerbe is independent from the SW map construction. We return to the SW map in the last section because it leads to the construction of nontrivial examples.

We also mention that related later work concerning noncommutative gerbes can be found in [6], [7], [8], [9], [10].

\section{SW map}

We denote by $a, \lambda$ and $\psi$ respectively the commutative $U(1)$ gauge potential, the Lie algebra valued gauge parameter and a charged matter field. We have the commutative field strenght $f=d a$ and the (infinitesimal) gauge transformations

$$
\begin{aligned}
& \delta_{\lambda} a=d \lambda \\
& \delta \psi=i \lambda \psi
\end{aligned}
$$

The noncommutative variables are denoted with capital letters $A, \Lambda \Psi$, the noncommutative field strength is given by $F_{\mu \nu}=\partial_{\mu} A_{v}-\partial_{\nu} A_{\mu}-i A_{\mu} \star A_{v}+i A_{v} \star A_{\mu}$ and the noncommutative gauge transformations read

$$
\begin{aligned}
& \hat{\delta}_{\Lambda} A=d \Lambda+i \Lambda \star A-i A \star \Lambda \\
& \hat{\delta}_{\Lambda} \Psi=i \Lambda \star \Psi
\end{aligned}
$$

Here the $\star$-product is the quantization of a given Poisson tensore $\theta$. For example if we are in $\mathbb{R}^{4}$ and we consider the Poisson tensor $\theta=\theta^{\mu v} \partial_{\mu} \otimes \partial_{v}$, with $\theta^{\mu v}$ constants, we obtain the Moyal star product $f \star g=f e^{\frac{i}{2} \hbar \theta^{\mu \nu} \overleftarrow{\partial}_{\mu} \vec{\partial}_{v}} g$.

The Seiberg-Witten map is a map between the commutative and the noncommutative potentials and gauge transformations [2]. It is found by requiring that (infinitesimal) commutative gauge transformations $\delta_{\lambda}$ correspond to noncommutative gauge transformations $\hat{\delta}_{\Lambda}$ :

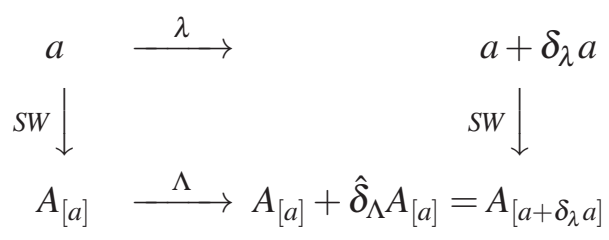

We are able to satisfy the equality $A_{[a]}+\hat{\delta}_{\Lambda} A_{[a]}=A_{\left[a+\delta_{\lambda} a\right]}$ because we allow $\Lambda$ to depend also on the gauge potential $a$ and its derivatives, i.e., $\Lambda=\Lambda_{[\lambda, a]}$. Of course both $A$ and $\Lambda$ depend on the Poisson tensor $\theta$ and the deformation parameter $\hbar$.

Commutativity of the above diagram implies that (at least locally) commutative and noncommutative gauge equivalence classes are in one-to-one correspondence. Since physics depends only 
on gauge equivalence classes it is then natural to expect that, provided the commutative and noncommutative lagrangians are suitably chosen, the same dynamics can be formulated using these two different gauge theories. This is for example what happens for the Dirac-Born-Infeld lagrangians describing the low energy (and slowly varying) fields on $D$-branes.

The noncommutative matter fields $\Psi$ can be similarly obtained by requiring [11, 12]

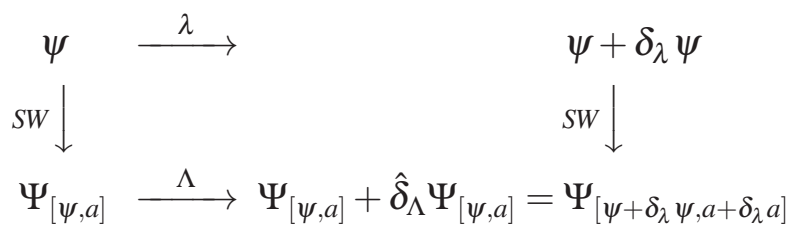

For constant and nondegenerate Poisson tensor $\theta^{\mu v}$, at first order we have (see the appendix of [13] for arbitrary $\theta$ )

$$
\begin{aligned}
A_{\rho} & =a_{\rho}+\frac{1}{2} \hbar \theta^{\mu v} a_{v} \partial_{\mu} a_{\rho}+\frac{1}{2} \hbar \theta^{\mu v} f_{\mu \rho} a_{v}+\mathscr{O}\left(\hbar^{2} \theta^{2}\right) \\
\Lambda & =\lambda+\frac{1}{2} \hbar \theta^{\mu v} \partial_{\mu} \lambda a_{v}+\mathscr{O}\left(\hbar^{2} \theta^{2}\right)
\end{aligned}
$$

The SW map can be worked out order by order in $\hbar$. Furthermore there is a beautiful relation between SW map and Kontsevich [14] construction of a $\star$-product associated with a Poisson tensor $\theta$ [15]. It allows to consider the SW map for arbitrary Poisson tensors and to show at all orders in $\hbar$ its existence.

In short in order to prove the Formality theorem Kontsevich introduced skew-symmetric multilinear maps $U_{n}$ (for $n=0,1,2 \ldots \infty$ ) that map tensor products of $n$ polyvector fields to multidifferential operators. These maps can in particular can be used to construct a $\star$-product (that is a bidifferential operator, $(f, g) \rightarrow f \star g)$ deformation quantization of a Poisson tensor $\theta=\theta^{\mu v}(x) \partial_{\mu} \otimes \partial_{v}$ (that is a bivector field).

These same maps can be used to construct the SW map, i.e., the noncommutative gauge potential and gauge transformation $(A, \Lambda)$ from the commutative ones $a, \lambda$.

\section{Covariantizing map $\mathscr{D}$}

A key point is played by the covariantizing map $\mathscr{D}$, see [3]. In gauge theory the notion of gauge potential $a$ (defined on an open $\mathrm{U}$ of the manifold $\mathrm{M}$ ) is equivalent to that of covariant derivative $D=d+a$; we recall that this name stems from the fact that if $\delta_{\lambda} \psi=i \lambda \psi$ then $D \psi$ transforms covariantly

$$
\delta_{\lambda} D \psi=i \lambda D \psi
$$

Similarly rather than considering the noncommutative gauge potential $A$ (defined on the open $U$ of $M$ ) we can consider the covariantizing map $\mathscr{D}$, a differential operator from the space of (formal power series in $\hbar$ of) smooth functions on the open $U$ of $M$ to itself,

$$
\mathscr{D}: C^{\infty}(U)[[\hbar]] \rightarrow C^{\infty}(U)[[\hbar]]
$$


If $\hat{\delta}_{\Lambda} \Psi=i \Lambda \star \Psi$ then in general $\hat{\delta}_{\Lambda} f \star \Psi=f \star \hat{\delta}_{\Lambda} \Psi \neq i \Lambda \star f \star \Psi$. The differential operator $\mathscr{D}$ covariantizes the function $f$ because it satisfies

$$
\hat{\delta}_{\Lambda} \mathscr{D}(f) \star \Psi=i \Lambda \star \mathscr{D}(f) \star \Psi .
$$

The Seiberg-Witten map can then be seen as a map between $a, \lambda$ and $\mathscr{D}, \Lambda$ where $\mathscr{D}$ depends on $a, \theta$ and their derivatives, we write $\mathscr{D}_{[a]}$, and similarly $\Lambda=\Lambda_{[\lambda, a]}$. The SW condition between commutative and noncommutative gauge transformations $A_{[a]}+\hat{\delta}_{\Lambda} A_{[a]}=A_{\left[a+\delta_{\lambda} a\right]}$ now reads, for all smooth functions $f \in C^{\infty}(U)[[\hbar]]$,

$$
\mathscr{D}_{[a]}(f)+\Lambda \star \mathscr{D}(f)-\mathscr{D}(f) \star \Lambda=\mathscr{D}_{\left[a+\delta_{\lambda} a\right]}(f) .
$$

The SW map $a \rightarrow \mathscr{D}_{[a]}, \lambda \rightarrow \Lambda_{[\lambda, a]}$ is constructed in [15]. There is of course an explicit relation between $\mathscr{D}$ and $A=A_{\mu} d x^{\mu}, \mathscr{D}\left(x^{\mu}\right)=x^{\mu}+\hbar \theta^{\mu v}(x) A_{v}$.

\section{Finite gauge transformations}

The infinitesimal gauge transformations can be integrated to finite ones, we thus obtain the usual commutative transformations

$$
\psi \stackrel{g}{\longrightarrow} \psi_{g}=g \psi
$$

and $a \stackrel{g}{\longrightarrow} a_{g}=a+i g d g^{-1}$ or equivalently

$$
D \stackrel{g}{\longrightarrow} D_{g}=g D g^{-1}
$$

and the corresponding noncommutative ones

$$
\begin{gathered}
\Psi_{[\psi, a]} \stackrel{g}{\longrightarrow} \Psi_{\left[\psi_{g}, a_{g}\right]}=G_{[g, a]} \star \Psi_{[\psi, a]} \\
\mathscr{D} \stackrel{g}{\longrightarrow} \mathscr{D}_{g}=A d_{\star G_{[g, a]}} \circ \mathscr{D}
\end{gathered}
$$

where, for all smooth functions $h \in C^{\infty}(U)[[\hbar]]$ that are $\star$-invertible ${ }^{1}$, the map $A d_{\star h}$ is defined by $A d_{\star h}(f)=h \star f \star h^{-1}$, for all $f \in C^{\infty}(U)[[\hbar]]$. Thus (2.14) equivalently reads, for all $f \in C^{\infty}(U)[[\hbar]]$,

$$
\mathscr{D}(f) \stackrel{g}{\longrightarrow} \mathscr{D}_{g}(f)=G_{[g, a]} \star \mathscr{D}(f) \star G_{[g, a]}^{-1} .
$$

\section{Noncommutative line bundles}

Let's apply two consecutive finite gauge transformations, with gauge group elements $g_{1}$ and $g_{2}$, they are the same as the gauge transformation with gauge group element $g_{1} g_{2}$,

$$
\Psi \stackrel{g_{1}}{\longrightarrow} G_{\left[g_{1}, a\right]} \star \Psi \stackrel{g_{2}}{\longrightarrow} G_{\left[g_{1}, a_{g_{2}}\right]} \star G_{\left[g_{2}, a\right]} \star \Psi=G_{\left[g_{1} g_{2}, a\right]} \star \Psi
$$

hence we obtain

$$
G_{\left[g_{1}, a_{g_{2}}\right]} \star G_{\left[g_{2}, a\right]}=G_{\left[g_{1} g_{2}, a\right]}
$$

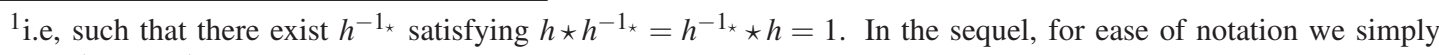
denote $h^{-1_{\star}}$ by $h^{-1}$ understanding that the inverse is taken with respect to the $\star$-product.
} 
Now let's consider $g_{1}$ and $g_{2}$ to be transition functions of a line bundle. This means that we have a smooth manifold $M$, and subordinate to an open cover $\left\{U^{i}\right\}$ of $M$ we have transition functions $g_{i j}: U^{i} \cap U^{j} \rightarrow U(1)$, that on triple overlaps $U^{i j k}=U^{i} \cap U^{j} \cap U^{k}$ satisfy $g_{i j} g_{j k}=g_{i k}$. Let also consider a connection on this line bundle, i.e., a set of 1-forms $\left\{a_{j}\right\}$ where each $a_{j}$ is defined on $U^{i}$, such that on double overlaps $a_{j}=a_{k}+i g_{i k} d g_{j k}^{-1}$.

If on $M$ we have also a Poisson structure $\theta$ we can construct in each open $U^{i}$ the corresponding covariantizing map $\mathscr{D}_{\left[a_{i}\right]}: C^{\infty}\left(U^{i}\right)[[\hbar]] \rightarrow C^{\infty}\left(U^{i}\right)[[\hbar]]$ associated (via SW map) to the connection $a^{i}$. Let $g_{1}=g_{i j}, g_{2}=g_{j k}, a=a_{k}$, then $a_{g_{2}}=a_{j}$; and we have $a_{j}=a_{k}+i g_{i k} d g_{j k}^{-1}$ in $U^{j k}=U^{i} \cap U^{j}$ and also $g_{1} g_{2}=g_{i j} g_{j k}=g_{i k}$ in $U^{i j k}$. Equation (3.2) then reads

$$
G_{\left[g_{i j}, a_{g_{J}}\right]} \star G_{\left[g_{j k}, a_{k}\right]}=G_{\left[g_{i k}, a_{k}\right]}
$$

that is the analogue of the line bundle cocycle condition $g_{i j} g_{j k}=g_{i k}$. This condition is complemented by the relation $\mathscr{D}^{i}=A d_{\star G^{i j}} \circ \mathscr{D}^{j}$, i.e.,

$$
A d_{\star G^{i j}}=\mathscr{D}^{i} \circ \mathscr{D}^{j^{-1}}
$$

Since the noncommutative structure emerging from (SW map) quantization of a commutative line bundle with connection is summarized in equations (3.3) and (3.4) we are led to define noncommutative line bundles as the structure given by the noncommutative transition functions $G^{i j}$ and covariantizing maps $\mathscr{D}$. We thus arrive at the following abstract definition (i.e. independent from the SW map construction followed so far) [4],

Definition Let $M$ be a smooth manifold with a $\star$-product (a bidifferential operator $\star: C^{\infty}(M)[[\hbar]] \otimes$ $C^{\infty}(M)[[\hbar]] \rightarrow C^{\infty}(M)[[\hbar]]$ that at zeroth order in $\hbar$ equals the usual pointwise product, and that is associative). Let 's consider a good covering $\left\{U^{i}\right\}$ of $M$. A noncommutative line bundle $\mathscr{L}$ is defined by a collection of $\mathbb{C}[[\hbar]]$-valued local transition functions $G^{i j} \in C^{\infty}\left(U^{i} \cap U^{j}\right)[[\hbar]]$, and a collection of maps $\mathscr{D}^{i}: C^{\infty}\left(U^{i}\right)[[\hbar]] \rightarrow C^{\infty}\left(U^{i}\right)[[\hbar]]$, formal power series in $\hbar$, starting with the identity, and with coefficients being differential operators, such that

$$
G^{i j} \star G^{j k}=G^{i k}
$$

on $U^{i} \cap U^{j} \cap U^{k}, G^{i i}=1$ on $U^{i}$, and

$$
\operatorname{Ad}_{\star} G^{i j}=\mathscr{D}^{i} \circ \mathscr{D}^{j-1}
$$

on $U^{i} \cap U^{j}$ or, equivalently, $\mathscr{D}^{i}(f) \star G^{i j}=G^{i j} \star \mathscr{D}^{j}(f)$ for all $f \in C^{\infty}\left(U^{i} \cap U^{j}\right)[[\hbar]]$.

Obviously, with this definition the local maps $\mathscr{D}^{i}$ can be used to define globally a new star product $\star^{\prime}$ (because the inner automorphisms $\mathrm{Ad}_{\star} G^{i j}$ do not affect $\star^{\prime}$ )

$$
\mathscr{D}^{i}\left(f \star^{\prime} g\right)=\mathscr{D}^{i} f \star \mathscr{D}^{i} g .
$$

We call the collection of maps $\mathscr{D}^{i}$ a global equivalence between the $\star$ and the $\star^{\prime}$ products globally defined on $M$. 
If on a Poisson Manifold $M$ we consider two equivalent commutative line bundles $L_{1}=\left\{g_{1}^{i j}\right\}$, $L_{2}\left\{g_{2}^{i j}\right\}$ with equivalent connections $\left\{a_{1}^{i}\right\}$ and $\left\{a_{2}^{i}\right\}$, and we apply the SW map we obtain an example of two equivalent noncommutative line bundles. In general (independently from the SW map construction) we say that two line bundles $\mathscr{L}_{1}=\left\{G_{1}^{i j}, \mathscr{D}_{1}^{i}, \star\right\}$ and $\mathscr{L}_{2}=\left\{G_{2}^{i j}, \mathscr{D}_{2}^{i}, \star\right\}$ are equivalent if there exists a collection of invertible local functions $H^{i} \in C^{\infty}=\left(U^{i}\right)[[\hbar]]$ such that

$$
G_{1}^{i j}=H^{i} \star G_{2}^{i j} \star\left(H^{j}\right)^{-1}
$$

and

$$
\mathscr{D}_{1}^{i}=\operatorname{Ad}_{\star} H^{i} \circ \mathscr{D}_{2}^{i} \cdot \mathscr{D}_{1}^{i}(.)=H^{i} \star \mathscr{D}_{2}^{i}(.) \star\left(H^{i}\right)^{-1} .
$$

The tensor product of two commutative line bundles has transition functions given by the products of the initial transition functions.

The tensor product of noncommutative line bundles $\mathscr{L}_{1}=\left\{G_{1}^{i j}, \mathscr{D}_{1}^{i}, \star_{1}\right\}$ and $\mathscr{L}_{2}=\left\{G_{2}^{i j}, \mathscr{D}_{2}^{i}, \star_{2}\right\}$ is well defined if $\star_{2}=\star_{1}^{\prime}$ (or $\star_{1}=\star_{2}^{\prime}$.) Then the corresponding tensor product is a line bundle $\mathscr{L}_{2} \otimes \mathscr{L}_{1}=\mathscr{L}_{21}=\left\{G_{12}^{i j}, \mathscr{D}_{12}^{i j}, \star_{1}\right\}$ defined as

$$
G_{12}^{i j}=\mathscr{D}_{1}^{i}\left(G_{2}^{i j}\right) \star_{1} G_{1}^{i j}=G_{1}^{i j} \star_{1} \mathscr{D}_{1}^{j}\left(G_{2}^{i j}\right)
$$

and

$$
\mathscr{D}_{12}^{i}=\mathscr{D}_{1}^{i} \circ \mathscr{D}_{2}^{i} \text {. }
$$

The order of indices of $\mathscr{L}_{21}$ shows the bimodule structure of the corresponding space of sections to be defined below, whereas the first index on the $G_{12}$ 's and $\mathscr{D}_{12}$ 's indicates the star product (here: $\star_{1}$ ) by which the objects multiply.

Let's recall that noncommutative manifolds are usually described by a noncommutative algebra $\mathscr{A}$ (that of would be complex valued functions on the manifold) and similarly, since finite projective modules are in one-to-one correspondence with vector bundles on a compact manifold $M$, noncommutative bundles are described as finite projective $\mathscr{A}$-modules. The noncommutative line bundle $\mathscr{L} \rightarrow M$, introduced in this section has an equivalent description as finite projective module. The module is that of sections $\Psi$ i.e., a collection of functions $\left\{\Psi^{i}\right\}, \Psi^{i} \in C^{\infty}\left(U^{i}\right)[[\hbar]]$ satisfying consistency relations

$$
\Psi^{i}=G^{i j} \star \Psi^{i}
$$

on all intersections $U^{i} \cap U^{j}$. The space of sections $\mathscr{E}$ is indeed a right $\mathscr{A}=\left(C^{\infty}(M)[[\hbar]], \star\right)$ module. We shall use the notation $\mathscr{E}_{\mathscr{A}}$ for it. The right action of the function $f \in \mathscr{A}$ is the regular one

$$
\Psi . f=\left\{\Psi^{k} \star f\right\} .
$$

Using the maps $\mathscr{D}^{i}$ it is easy to turn $\mathscr{E}$ also into a left $\mathscr{A}^{\prime}=\left(C^{\infty}(M)[[\hbar]], \star^{\prime}\right)$ module $\mathscr{A}^{\prime} \mathscr{E}$. The left action of $\mathscr{A}^{\prime}$ is given by

$$
f . \Psi=\left\{\mathscr{D}^{i}(f) \star \Psi^{i}\right\} .
$$

It is easy to check, using (3.6), that the left action (3.14) is compatible with (3.12). From the property (3.7) of the maps $\mathscr{D}^{i}$ we find

$$
f .(g . \Psi)=\left(f \star^{\prime} g\right) . \Psi .
$$


Together we have a bimodule structure $\mathscr{A}^{\prime} \mathscr{E}_{\mathscr{A}}$ on the space of sections. It can be shown that the two $\star$ products $\star$ and $\star^{\prime}$ are Morita equivalent [4].

\section{Commutative gerbes}

A (complex) line bundle can be represented by a 1-cocycle in Čech cohomology, i.e., a collection of smooth complex valued (or $U(1)$-valued) transition functions $g^{\alpha \beta}$ on the intersections $U_{\alpha} \cap U_{\beta}$ of an open cover $\left\{U_{\alpha}\right\}$ of a manifold $M$ satisfying $g_{\alpha \beta}=g_{\beta \alpha}^{-1}$, and $g_{\alpha \beta} g_{\beta \gamma} g_{\gamma \alpha}=1$ on $U_{\alpha} \cap U_{\beta} \cap U_{\gamma}$. Similarly, an abelian gerbe can be represented by a 2-cocycle in Čech cohomology, i.e., by a collection $\lambda=\left\{\lambda_{\alpha \beta \gamma}\right\}$ of maps $\lambda_{\alpha \beta \gamma}: U_{\alpha} \cap U_{\beta} \cap U_{\gamma} \rightarrow U(1)$, valued in the abelian group $U(1)$, satisfying

and the 2-cocycle condition

$$
\lambda_{\alpha \beta \gamma}=\lambda_{\beta \alpha \gamma}^{-1}=\lambda_{\alpha \gamma \beta}^{-1}=\lambda_{\gamma \beta \alpha}^{-1}
$$

$$
\delta \lambda=\lambda_{\beta \gamma \delta} \lambda_{\alpha \gamma \delta}^{-1} \lambda_{\alpha \beta \delta} \lambda_{\alpha \beta \gamma}^{-1}=1
$$

on $U_{\alpha} \cap U_{\beta} \cap U_{\gamma} \cap U_{\delta}$. The collection $\lambda=\left\{\lambda_{\alpha \beta \gamma}\right\}$ of maps with the stated properties represents a gerbe in the same sense as a collection of transition functions represents a line bundle. In the special case where $\lambda$ is a Čech 2-coboundary with $\lambda=\delta h$, i.e., $\lambda_{\alpha \beta \gamma}=h_{\alpha \beta} h_{\beta \gamma} h_{\gamma \alpha}$, we say that the collection $h=\left\{h_{\alpha \beta}\right\}$ of functions $h_{\alpha \beta}: U_{\alpha} \cap U_{\beta} \rightarrow U(1)$ represents a trivialization of a gerbe.

There exists a local trivialization of a 2-cocycle for any particular open set $U_{0}$ of the covering: defining $h_{\beta \gamma} \equiv \lambda_{0 \beta \gamma}$ with $\beta, \gamma \neq 0$ we find from the 2-cocycle condition that $\lambda_{\alpha \beta \gamma}=h_{\alpha \beta} h_{\beta \gamma} h_{\gamma \alpha}$. In particular on double overlaps (say $U_{0} \cap U_{0^{\prime}}$ ) we have two trivializations $\left\{h_{\alpha \beta}\right\}$ and $\left\{h_{\alpha \beta}^{\prime}\right\}$. Now we notice that the ratio $g_{\alpha \beta} \equiv h_{\alpha \beta} / h_{\alpha \beta}^{\prime}$ of two 2-coboundaries $\left\{h_{\alpha \beta}\right\},\left\{h_{\alpha \beta}^{\prime}\right\}$ representing two trivializations of a gerbe is a 1-cocycle: $g_{\alpha \beta} g_{\beta \gamma} g_{\gamma \alpha}=1$. This observation leads to a definition of an abelian gerbe (more precisely "gerbe data") á la Hitchin [5] in terms of line bundles on the double overlaps of the cover. Thus while a line bundle is characterized by transition functions on double oberlaps $U_{\alpha} \cap U_{\beta}$, a gerbe is characterized by transition line bundles on double overlaps $U_{\alpha} \cap U_{\beta}$. A gerbe á la Hitchin is then a collection of line bundles $L_{\alpha \beta}$ for each double overlap $U_{\alpha} \cap U_{\beta}$, such that:

G1 There is an isomorphism $L_{\alpha \beta} \cong L_{\beta \alpha}^{-1}$.

G2 There is a trivialization $\lambda_{\alpha \beta \gamma}$ of $L_{\alpha \beta} \otimes L_{\beta \gamma} \otimes L_{\gamma \alpha}$ on $U_{\alpha} \cap U_{\beta} \cap U_{\gamma}$.

G3 The trivialization $\lambda_{\alpha \beta \gamma}$ satisfies $\delta \lambda=1$ on $U_{\alpha} \cap U_{\beta} \cap U_{\gamma} \cap U_{\delta}$.

\section{Noncommutative gerbes}

Since gerbes can be defined via line bundles and their products, and since in Section 3 we have noncommutative line bundles and we can also consider their product we arrive at the following

Definition Consider a manifold $M$, a covering $\left\{U_{\alpha}\right\}$ (not necessarily a good one) and on each local patch $U_{\alpha}$ a star product $\star_{\alpha}$. Let also consider a good covering $\left\{U_{\alpha \beta}^{i}\right\}$ of each double intersection $U_{\alpha} \cap U_{\beta}$ and a noncommutative line bundle on $U_{\alpha} \cap U_{\beta}, \mathscr{L}_{\beta \alpha}=\left\{G_{\alpha \beta}^{i j}, \mathscr{D}_{\alpha \beta}^{i}, \star_{\alpha}\right\}$

$$
G_{\alpha \beta}^{i j} \star_{\alpha} G_{\alpha \beta}^{j k}=G_{\alpha \beta}^{i k}, \quad G_{\alpha \beta}^{i i}=1,
$$




$$
\mathscr{D}_{\alpha \beta}^{i}(f) \star_{\alpha} G_{\alpha \beta}^{i j}=G_{\alpha \beta}^{i j} \star_{\alpha} \mathscr{D}_{\alpha \beta}^{j}(f)
$$

We require

$$
\mathscr{D}_{\alpha \beta}^{i}\left(f \star_{\beta} g\right)=\mathscr{D}_{\alpha \beta}^{i}(f) \star_{\alpha} \mathscr{D}_{\alpha \beta}^{i}(g),
$$

i.e, we require the space of sections of this line bundle to be a left module with respect to the algebra $\mathscr{A}_{\beta}=\left(C^{\infty}\left(U^{\alpha} \cap U^{\beta}\right)[[\hbar]], \star_{\beta}\right)$ and a right module with respect to the algebra $\mathscr{A}_{\alpha}=\left(C^{\infty}\left(U^{\alpha} \cap\right.\right.$ $\left.\left.U^{\beta}\right)[[\hbar]], \star_{\alpha}\right)^{2}$. The line bundles $\left\{\mathscr{L}_{\beta \alpha}\right\}$ define a noncommutative gerbe if

G1 $\mathscr{L}_{\alpha \beta}=\left\{G_{\beta \alpha}^{i j}, \mathscr{D}_{\beta \alpha}^{i}, \star_{\beta}\right\}$ and $\mathscr{L}_{\beta \alpha}=\left\{G_{\alpha \beta}^{i j}, \mathscr{D}_{\alpha \beta}^{i}, \star_{\alpha}\right\}$ are related as follows

$$
\left\{G_{\beta \alpha}^{i j}, \mathscr{D}_{\beta \alpha}^{i}, \star_{\beta}\right\}=\left\{\left(\mathscr{D}_{\alpha \beta}^{j}\right)^{-1}\left(G_{\alpha \beta}^{j i}\right),\left(\mathscr{D}_{\alpha \beta}^{i}\right)^{-1}, \star_{\beta}\right\}
$$

i.e. $\mathscr{L}_{\alpha \beta}=\mathscr{L}_{\beta \alpha}^{-1}$. (Notice also that $\left(\mathscr{D}_{\alpha \beta}^{j}\right)^{-1}\left(G_{\alpha \beta}^{j i}\right)=\left(\mathscr{D}_{\alpha \beta}^{i}\right)^{-1}\left(G_{\alpha \beta}^{j i}\right)$.)

G2 $2_{\star}$ On the triple intersection $U_{\alpha} \cap U_{\beta} \cap U_{\gamma}$ the tensor product $\mathscr{L}_{\gamma \beta} \otimes \mathscr{L}_{\beta \alpha}$ is equivalent to the line bundle $\mathscr{L}_{\gamma \alpha}$. Explicitly

$$
\begin{aligned}
G_{\alpha \beta}^{i j} \star_{\alpha} \mathscr{D}_{\alpha \beta}^{j}\left(G_{\beta \gamma}^{i j}\right) & =\Lambda_{\alpha \beta \gamma}^{i} \star_{\alpha} G_{\alpha \gamma \star}^{i j}\left(\Lambda^{j}\right)_{\alpha \beta \gamma}^{-1}, \\
\mathscr{D}_{\alpha \beta}^{i} \circ \mathscr{D}_{\beta \gamma}^{i} & =\operatorname{Ad}_{\star_{\alpha}} \Lambda_{\alpha \beta \gamma}^{i} \circ \mathscr{D}_{\alpha \gamma}^{i} .
\end{aligned}
$$

$\mathbf{G 3}_{\star}$ On the quadruple intersection $U_{\alpha} \cap U_{\beta} \cap U_{\gamma} \cap U_{\delta}$

$$
\begin{gathered}
\Lambda_{\alpha \beta \gamma}^{i} \star_{\alpha} \Lambda_{\alpha \gamma \delta}^{i}=\mathscr{D}_{\alpha \beta}^{i}\left(\Lambda_{\beta \gamma \delta}^{i}\right) \star_{\alpha} \Lambda_{\alpha \beta \delta}^{i}, \\
\Lambda_{\alpha \beta \gamma}^{i}=\left(\Lambda_{\alpha \gamma \beta}^{i}\right)^{-1} \quad \text { and } \quad \mathscr{D}_{\alpha \beta}^{i}\left(\Lambda_{\beta \gamma \alpha}^{i}\right)=\Lambda_{\alpha \beta \gamma}^{i} .
\end{gathered}
$$

The cocycle condition (5.7) is consistent with conditions $\mathbf{G} \mathbf{1}_{\star}$ and $\mathbf{G} \boldsymbol{2}_{\star}$, indeed, define

$$
\mathscr{D}_{\alpha \beta \gamma}^{i}=\mathscr{D}_{\alpha \beta}^{i} \circ \mathscr{D}_{\beta \gamma}^{i} \circ \mathscr{D}_{\gamma \alpha}^{i} .
$$

Then it is easy to see that

$$
\mathscr{D}_{\alpha \beta \gamma}^{i} \circ \mathscr{D}_{\alpha \gamma \delta}^{i} \circ \mathscr{D}_{\alpha \delta \beta}^{i}=\mathscr{D}_{\alpha \beta}^{i} \circ \mathscr{D}_{\beta \gamma \delta}^{i} \circ \mathscr{D}_{\beta \alpha}^{i}
$$

In view of (5.6) this implies that

$$
\Lambda_{\alpha \beta \gamma \delta}^{i} \equiv \mathscr{D}_{\alpha \beta}^{i}\left(\Lambda_{\beta \gamma \delta}^{i}\right) \star_{\alpha} \Lambda_{\alpha \beta \delta}^{i} \star_{\alpha} \Lambda_{\alpha \delta \gamma}^{i} \star_{\alpha} \Lambda_{\alpha \gamma \beta}^{i}
$$

is central. Using this and the associativity of $\star_{\alpha}$ together with (5.5) applied to the triple tensor product $\mathscr{L}_{\delta \gamma} \otimes \mathscr{L}_{\gamma \beta} \otimes \mathscr{L}_{\beta \alpha}$ transition functions

$$
G_{\alpha \beta \gamma}^{i j} \equiv G_{\alpha \beta}^{i j} \star_{\alpha} \mathscr{D}_{\alpha \beta}^{j}\left(G_{\beta \gamma}^{i j}\right) \star_{\alpha} \mathscr{D}_{\alpha \beta}^{j}\left(\mathscr{D}_{\beta \gamma}^{j}\left(G_{\gamma \delta}^{i j}\right)\right)
$$

\footnotetext{
${ }^{2}$ The opposite order of indices labelling the line bundles and the corresponding transition functions $G$ and maps $\mathscr{D}$ simply reflects a choice of convention. As in Section 3 the order of indices of $\mathscr{L}_{\alpha \beta}$ indicates the bimodule structure of the corresponding space of sections, whereas the order of Greek indices on $G$ 's and $\mathscr{D}$ 's indicates the star product in which the objects multiply. The product always goes with the first index of the multiplied objects
} 
reveals that $\Lambda_{\alpha \beta \gamma \delta}^{i}$ is independent of $i$. It is therefore consistent to set $\Lambda_{\alpha \beta \gamma \delta}^{i}$ equal to 1. A similar consistency check works also for (5.8).

Let us consider the triple tensor product $\mathscr{L}_{\alpha \gamma \beta} \equiv \mathscr{L}_{\alpha \gamma} \otimes \mathscr{L}_{\gamma \beta} \otimes \mathscr{L}_{\beta \alpha}$ with maps $\mathscr{D}_{\alpha \beta \gamma}^{i}$ and transition functions (5.11). Using condition $\mathbf{G} \mathbf{1}_{\star}$ and $\mathbf{G} \mathbf{2}_{\star}$ one can show that the product bundle

$$
\mathscr{L}_{\alpha \beta \gamma \delta}=\mathscr{L}_{\alpha \beta \gamma} \otimes \mathscr{L}_{\alpha \gamma \delta} \otimes \mathscr{L}_{\alpha \delta \beta} \otimes \mathscr{L}_{\alpha \beta} \otimes \mathscr{L}_{\beta \delta \gamma} \otimes \mathscr{L}_{\beta \alpha}
$$

is canonically trivial: it is a product of canonically trivial bundles of the kind $\mathscr{L}_{\alpha \beta}{ }^{-1} \mathscr{L}_{\alpha \beta}$. These bundles have canonical unit section and hence also $\mathscr{L}_{\alpha \beta \gamma \delta}$ has canoncial unit section. Moreover using $\mathbf{G} \boldsymbol{2}_{\star}$ one can show that $\mathscr{L}_{\alpha \beta \gamma \delta}$ has also transition functions $G_{\alpha \beta \gamma \delta}^{i j}=1$, maps $\mathscr{D}_{\alpha \beta \gamma \delta}^{i}=$ id and global section $\left(\Lambda_{\alpha \beta \gamma \delta}^{i}\right)$. The cocycle condition (5.7) implies $\left(\Lambda_{\alpha \beta \gamma \delta}^{i}\right)$ to be the canonical unit section. If two of the indices $\alpha, \beta, \gamma, \delta$ are equal, triviality of the bundle $\mathscr{L}_{\alpha \beta \gamma \delta}$ implies (5.8).

The noncommutatve gerbe definition simplifies if we consider $\left\{U_{\alpha}\right\}$ to be a good covering (this is always doable by refining the initial covering). Then the covering $\left\{U_{\alpha \beta}^{i}\right\}$ of $U_{\alpha} \cap U_{\beta}$ can be composed just by the open $U_{\alpha} \cap U_{\beta}$ so that the bundles $\mathscr{L}_{\beta \alpha}=\left\{G_{\alpha \beta}^{i j}, \mathscr{D}_{\alpha \beta}^{i}, \star_{\alpha}\right\}$ simply read $\mathscr{L}_{\beta \alpha}=\left\{\mathscr{D}_{\alpha \beta}, \star_{\alpha}\right\}$. In this case $\Lambda_{\alpha \beta \gamma}$ is a global function on the triple intersection $U_{\alpha} \cap U_{\beta} \cap U_{\gamma}$, and on the quadruple overlap $U_{\alpha} \cap U_{\beta} \cap U_{\gamma} \cap U_{\delta}$ it satisfies conditions analogous to (5.7) and (5.8)

$$
\begin{gathered}
\Lambda_{\alpha \beta \gamma} \star_{\alpha} \Lambda_{\alpha \gamma \delta}=\mathscr{D}_{\alpha \beta}\left(\Lambda_{\beta \gamma \delta}\right) \star_{\alpha} \Lambda_{\alpha \beta \delta}, \\
\Lambda_{\alpha \beta \gamma}=\left(\Lambda_{\alpha \gamma \beta}\right)^{-1} \quad \text { and } \quad \mathscr{D}_{\alpha \beta}\left(\Lambda_{\beta \gamma \alpha}\right)=\Lambda_{\alpha \beta \gamma} .
\end{gathered}
$$

Also

$$
\mathscr{D}_{\alpha \beta} \circ \mathscr{D}_{\beta \gamma} \circ \mathscr{D}_{\gamma \alpha}=\operatorname{Ad}_{\star \alpha} \Lambda_{\alpha \beta \gamma} .
$$

So we can take formulas (5.13)-(5.15) as a definition of a noncommutative gerbe in the case of a good covering $\left\{U_{\alpha}\right\}$. We say that the gerbe is defined by the local data $\left\{\star_{\alpha}, \mathscr{D}_{\alpha \beta}, \Lambda_{\alpha \beta \gamma}\right\}$.

From now on we shall consider only good coverings. A noncommutative gerbe defined by $\left\{\star_{\alpha}, \mathscr{D}_{\alpha \beta}, \Lambda_{\alpha \beta \gamma}\right\}$ is said to be trivial if there exists a global star product $\star$ on $M$ and a collection of maps $\mathscr{D}_{\alpha}$ that provide a local equivalence between the global product $\star$ and the local products $\star \alpha$, i.e,

$$
\mathscr{D}_{\alpha}(f) \star \mathscr{D}_{\alpha}(g)=\mathscr{D}_{\alpha}\left(f \star_{\alpha} g\right)
$$

and that satisfy the following two conditions:

$$
G_{\alpha \beta} \star G_{\beta \gamma}=\mathscr{D}_{\alpha}\left(\Lambda_{\alpha \beta \gamma}\right) \star G_{\alpha \gamma}
$$

and

$$
\operatorname{Ad}_{\star} G_{\alpha \beta} \circ \mathscr{D}_{\beta}=\mathscr{D}_{\alpha} \circ \mathscr{D}_{\alpha \beta}
$$

where $G_{\alpha \beta}$ are a collection of "twisted" (by $\mathscr{D}_{\alpha}\left(\Lambda_{a l \beta \gamma}\right)$ ) transition functions, defined on each over$\operatorname{lap} U_{\alpha} \cap U_{\beta}$.

Locally, every noncommutative gerbe is trivial as is easily seen from (5.13), (5.14) and (5.15) by fixing the index $\alpha$. 
More in general two noncommutative gerbes respectively defined ${ }^{3}$ by their corresponding local data $\left\{\star_{\alpha}, \mathscr{D}_{\alpha \beta}, \Lambda_{\alpha \beta \gamma}\right\}$ and $\left\{\star_{\alpha}^{\prime}, \mathscr{D}_{\alpha \beta}^{\prime}, \Lambda_{\alpha \beta \gamma}^{\prime}\right\}$ are equivalent if there exist local equivalences $\mathscr{D}_{\alpha}$ of star products $\star_{\alpha}$ and $\star_{\alpha}^{\prime}$, i.e.,

$$
\mathscr{D}_{\alpha}(f) \star_{\alpha}^{\prime} \mathscr{D}_{\alpha}(g)=\mathscr{D}_{\alpha}\left(f \star_{\alpha} g\right)
$$

and local functions $\Lambda_{\alpha \beta}$ such that

$$
\operatorname{Ad}_{\star_{\alpha}^{\prime}} \Lambda_{\alpha \beta} \circ \mathscr{D}_{\alpha \beta}^{\prime} \circ \mathscr{D}_{\beta}=\mathscr{D}_{\alpha} \circ \mathscr{D}_{\alpha \beta}
$$

and

$$
\mathscr{D}_{\alpha}\left(\Lambda_{\alpha \beta \gamma}\right) \star_{\alpha}^{\prime} \Lambda_{\alpha \gamma}=\Lambda_{\alpha \beta} \star_{\alpha}^{\prime} \mathscr{D}_{\alpha \beta}^{\prime}\left(\Lambda_{\beta \gamma}\right) \star_{\alpha}^{\prime} \Lambda_{\alpha \beta \gamma}^{\prime}
$$

We conclude this section with the following remarks concerning the role of local functions $\Lambda_{\alpha \beta \gamma}$ and $\mathscr{D}_{\alpha \beta}$ satisfying relations (5.13)-(5.15). These represent an honest non-abelian 2-cocycle, as defined for example in [16]. It follows from the discussion of Section 2, that each $\mathscr{D}_{\alpha \beta}$ defines an equivalence, in the sense of deformation quantization, of star products $\star_{\alpha}$ and $\star_{\beta}$ on $U_{\alpha} \cap U_{\beta}$. The non-triviality of the non-abelian 2-cocycle (5.13)-(5.15) can therefore be seen as an obstruction to gluing the collection of local star products $\left\{\star_{\alpha}\right\}$, i.e., the collection of local rings $C^{\infty}\left(U_{\alpha}\right)[[\hbar]]$, into a global one. We also mention that in [17] a 2-cocycle similar to that of (5.13)-(5.15) represents an obstruction to gluing together certain local rings appearing in quantization of contact manifolds.

By the correspondence (in the sense of 2-categories, see [16] for details) between degree two non-abelian cohomology classes and equivalence classes of (standard) gerbes understood as locally non-empty and locally connected stack in groupoids there is such a (standard) gerbe corresponding to this specific non-abelian 2-cocycle. Hence our definition of a noncommutative gerbe leads to a non-abelian gerbe in the standard sense of Giraud, Deligne, Breen and Brylinski [18, 19, 16, 20].

\section{Noncommutative gerbes from quantization of twisted Poisson structures}

Consider a closed integral 3-form on $M, H \in H^{3}(M, \mathbb{Z})$. Such a form is known to define a gerbe on $M$. We can find a good covering $\left\{U_{\alpha}\right\}$ and local potentials $B_{\alpha}$ with $H=d B_{\alpha}$ for $H$. On $U_{\alpha} \cap U_{\beta}$ the difference of the two local potentials $B_{\alpha}-B_{\beta}$ is closed and hence exact: $B_{\alpha}-B_{\beta}=d a_{\alpha \beta}$. On a triple intersection $U_{\alpha} \cap U_{\beta} \cap U_{\gamma}$ we have

$$
a_{\alpha \beta}+a_{\beta \gamma}+a_{\gamma \alpha}=-i \lambda_{\alpha \beta \gamma} d \lambda_{\alpha \beta \gamma}^{-1} .
$$

The collection of local functions $\left\{\lambda_{\alpha \beta \gamma}\right\}$ represents a gerbe.

Let us also consider on $M$ an antisymmetric bivector field $\theta=\theta^{(0)}+\hbar \theta^{(1)}+\ldots$ (each coefficient of the power series in $\hbar$ being an antisymmetric vector field). We call $\theta$ a formal bivector field. Let $\theta$ be such that

$$
[\theta, \theta]=\hbar \theta^{*} H
$$

where $[$,$] is the Schouten-Nijenhuis bracket and \theta^{*}$ denotes the natural map sending $n$-forms to $n$-vector fields by "using $\theta$ to raise indices". Explicitly, in local coordinates, $\left(\theta^{*} H\right)^{i j k}=$

\footnotetext{
${ }^{3}$ after passing to a common refinement of respective trivializing coverings, if necessary
} 
$\theta^{i m} \boldsymbol{\theta}^{\text {jn }} \boldsymbol{\theta}^{k o} H_{m n o}$. We call $\theta$ a Poisson structure twisted by $H[21,22,23]$. Indeed when $H \rightarrow 0$ we recover a true Poisson structure. On each $U_{\alpha}$ we can introduce a local formal Poisson structure

$$
\theta_{\alpha}=\theta\left(1-\hbar B_{\alpha} \theta\right)^{-1}
$$

indeed $\left[\theta_{\alpha}, \theta_{\alpha}\right]=0$. Explicitly, using local coordinates $\theta_{\alpha}^{i j}=\theta^{i k}\left(\left(1-\hbar B_{\alpha} \theta\right)^{-1}\right)_{k}^{j}$ with $(1-$ $\left.\hbar B_{\alpha} \theta\right)_{k}^{j}=\delta_{k}{ }^{j}-\hbar B_{\alpha k l} \theta^{l j}$. The Poisson structures $\theta_{\alpha}$ and $\theta_{\beta}$ are related on the intersection $U_{\alpha} \cap U_{\beta}$ by

$$
\theta_{\alpha}=\theta_{\beta}\left(1+\hbar F_{\beta \alpha} \theta_{\beta}\right)^{-1},
$$

with an exact $F_{\beta \alpha}=d a_{\beta \alpha}$. Now we can use Kontsevich's formality [14] to obtain local star products $\star_{\alpha}$ and to construct for each intersection $U_{\alpha} \cap U_{\beta}$ the corresponding equivalence maps $\mathscr{D}_{\alpha \beta}$ between $\star_{\alpha}$ and $\star_{\beta}$. See [15, 4] for an explicit formula for the equivalence maps. According to our discussion in the previous section these $\mathscr{D}_{\alpha \beta}$, supplemented by trivial transition functions, define a collection of trivial line bundles $\mathscr{L}_{\beta \alpha}$. On each triple intersection we then have

$$
\mathscr{D}_{\alpha \beta} \circ \mathscr{D}_{\beta \gamma} \circ \mathscr{D}_{\gamma \alpha}=\operatorname{Ad}_{\star_{\alpha}} \Lambda_{\alpha \beta \gamma} .
$$

It follows from the discussion after formula (5.8) that the collection of local functions $\left\{\Lambda_{\alpha \beta \gamma}\right\}$ represents a noncommutative gerbe (a deformation quantization of the classical gerbe represented by $\left.\left\{\lambda_{\alpha \beta \gamma}\right\}\right)$ if each of the central functions $\Lambda_{\alpha \beta \gamma \delta}$ introduced there can be chosen to be equal to 1 . See [24, section 5] and [25] that this is really the case. As mentioned at the end of the previous section, the non-triviality of the non-abelian 2-cocycle (5.13)-(5.15) can be seen as an obstruction to gluing the collection of local star products $\left\{\star_{\alpha}\right\}$, i.e., the collection of local rings $C^{\infty}\left(U_{\alpha}\right)[[\hbar]]$, into a global one. Hence, in the context of this section, this obstruction comes as a deformation quantization of the classical obstruction to gluing together local formal Poisson brackets $\{,\}_{\alpha}$ into a global one.

\section{References}

[1] P. Aschieri, I. Baković, B. Jurco and P. Schupp, Noncommutative gerbes and deformation quantization, J. Geom. Phys., 60, 1754 (2010), [hep-th/0206101].

[2] N. Seiberg and E. Witten, String theory and noncommutative geometry, JHEP 9909, 032 (1999), [hep-th/9908142].

[3] J. Madore, S. Schraml, P. Schupp and J. Wess, Gauge theory on noncommutative spaces, Eur. Phys. J. C 16, 161 (2000), [hep-th/0001203].

[4] B. Jurčo, P. Schupp and J. Wess, "Noncommutative line bundle and Morita equivalence," Lett. Math. Phys. 61, 171-186 (2002), [arXiv:hep-th/0106110]

[5] N. Hitchin, "Lectures on special Lagrangian submanifolds," in "Winter School on Mirror Symmetry, Vector Bundles and Lagrangian Submanifolds," C. Vafa and S.-T. Yau (eds.), Studies in Advanced Mathematics 23, AMS/International Press, Providence (2001), 151 - 182; [arXiv:math.dg/9907034]

[6] P. Bressler, A. Gorokhovsky, R. Nest, B. Tsygan, "Deformation quantization of gerbes," Adv. Math. 214, 230-266 (2007), arXiv:math/0512136; P. Bressler, A. Gorokhovsky, R. Nest, B. Tsygan, "Deformations of algebroid stacks," arXiv:0810.0030; P. Bressler, A. Gorokhovsky, R. Nest, B. Tsygan, "Formality for algebroid stacks," arXiv:0903.1841 
[7] J. Mickelsson, “Gerbes on quantum groups,” e-Print: math/0308235

[8] F. Petalidou, “On the geometric quantization of twisted Poisson manifolds," J. Math. Phys. 48, 083502 (2007)

[9] M. Kashiwara, P. Schapira, "Deformation quantization modules I:Finiteness and duality," arXiv:0802.1245; "Deformation quantization modules II. Hochschild class” arXiv:0809.4309

[10] A. D’Agnolo, P. Polesello, “Algebroid stacks and WKB Operators,” in: Microlocal Analysis and Asymptotic Analysis, RIMS Kokyuroku, Kyoto Univ. 1397 (2004), 118-125

[11] B. Jurčo, S. Schraml, P. Schupp and J. Wess, Enveloping algebra valued gauge transformations for non-Abelian gauge groups on non-commutative spaces, Eur. Phys. J. C17, 521 (2000), [hep-th/0006246].

[12] B. Jurčo, L. Möller, S. Schraml, P. Schupp and J. Wess, Construction of non-Abelian gauge theories on noncommutative spaces, Eur. Phys. J. C21, 383 (2001), [hep-th/0104153 ].

[13] P. Aschieri, B. Jurčo, P. Schupp and J. Wess, Noncommutative GUTs, standard model and C, P, T, Nucl. Phys. B 651, 45 (2003), [hep-th/0205214].

[14] M. Kontsevich, “Deformation quantization of Poisson manifolds, I,” Lett. Math. Phys. 66, 157-216 (2003), [arXiv:q-alg/9709040]

[15] B. Jurčo, P. Schupp and J. Wess, "Noncommutative gauge theory for Poisson manifolds," Nucl. Phys. B 584, 784 (2000), [arXiv:hep-th/0005005];

"Nonabelian noncommutative gauge theory via noncommutative extra dimensions," Nucl. Phys. B 604, 148 (2001), [arXiv:hep-th/0102129]

[16] L. Breen, “On the classification of 2-gerbes and 2-stacks,” Astérisque 225 (1994)

[17] M. Kashiwara, "Quantization of contact manifolds” Publ. Res. Inst. Math. Sci. 32, 1 (1996).

[18] J. Giraud, “Cohomologie non-abélienne," Grundlehren der mathematischen Wissenschaften, Band 179, Springer Verlag, Berlin (1971).

[19] P. Deligne and J. S. Milne, “Tannakian Categories,” Lecture Notes in Mathematics 900, 101-228, Springer-Verlag (1981)

[20] J. L. Brylinski, “Loop Spaces, Characteristic Classes And Geometric Quantization,” Progress in mathematics 107, Birkhaeuser, Boston, USA (1993)

[21] P. Ševera and A. Weinstein, "Poisson geometry with a 3-form background," Prog. Theor. Phys. Suppl. 144, 145-154 (2001), [arXiv:math.sg/0107133]

[22] J. S. Park, "Topological open p-branes," in "Seoul 2000, Symplectic geometry and mirror symmetry," 311-384; [arXiv:hep-th/0012141]

[23] C. Klimčík and T. Strobl, “WZW-Poisson manifolds,” J. Geom. Phys. 43, 341 Ű344 (2002), [arXiv:math.sg/0104189]

[24] P. Ševera, "Quantization of Poisson Families and of twisted Poisson structures," Lett. Math. Phys. 63 (2003), [arXiv:math.qa/0205294]

[25] M. Kontsevich, “Deformation quantization of algebraic varietes,” Lett. Math. Phys. 56, 271 (2001), [arXiv:math.ag/0106006] 